\title{
Management of Hypoglycemia in Patients with Type 2 Diabetes
}

\author{
Jeff Unger* \\ Director, Unger Primary Care Concierge Medical Group, Rancho Cucamonga, USA
}

Received: 畊 September 19, 2018; Published: 制 September 24, 2018

*Corresponding author: Jeff Unger, Director, Unger Primary Care Concierge Medical Group, Rancho Cucamonga, USA

\section{Introduction}

The rate limiting step of intensive diabetes management for patients with diabetes is treatment induced hypoglycemia. Thus, hypoglycemia remains a significant barrier in optimizing glycemic control and reducing long-term diabetes related complications including cardiovascular death, stroke, and all cause-mortality [1]. Cardiovascular (CV) events are the leading cause of death among patients with diabetes. The risk of $\mathrm{CV}$ death is twice that of patients without diabetes [2]. Severe hypoglycemia (resulting in cognitive impairment) can occur across a broad spectrum of A1C levels. Patients achieving near-normal glycemia $(<6 \%)$ and those who were poorly controlled $(\geq 9 \%)$ appeared to be at the highest risk for severe hypoglycemia [3]. HbA1c and risk of severe hypoglycemia in type 2 diabetes. The Diabetes and Aging Study. Diabetes Care. 12013; 36 (11): 3535-3542) Hypoglycemia triggers a vascular and inflammatory cascade which can result in vascular constriction, tachycardia, thrombosis, and fatal arrythmias [3]. Therefore, patients who have existing CV disease should have their glycemic targets relaxed to mitigate the risk of hypoglycemia. Recurrent hypoglycemia lowers or even eliminates the blood glucose concentration threshold at which patients develop a sympathetic response likely to prompt them to take evasive action in time to reverse an impending event. Elderly patients may lose their balance without warning, falling to the ground. The subsequent confusion after such a fall is likely to be attributed to "the aging process" rather than to deficient glucose counter regulation.

Clinicians should remind patients with diabetes who use insulin to monitor their blood glucose levels prior to operating a motor vehicle. The single most significant factor associated with driving collisions for drivers with diabetes appears to be a recent history of severe hypoglycemia, regardless of the type of diabetes or the treatment used [4]. A single hypoglycemia event can deplete counterregulatory hormone levels which favor recognition and reversal of low plasma glucose, resulting in a lack of awareness of a hypoglycemic event. Nocturnal hypoglycemia may result in patients arriving late to work or missing entire days at the office. Patients who experience hypoglycemia are likely to use extra test strips for fear of having a recurrence of low blood glucose levels. Calls to doctors for guidance on glucose management are increased after an episode of hypoglycemia, and patients often inappropriately self-titrate their medications to avoid future events [5]. Hypoglycemia increases the risk of cardiovascular and allcause mortality in patients with diabetes [6]. Severe hypoglycemia is associated with a macrovascular events hazards ratio (HR) of 2.88 and a microvascular events HR of 1.81. The mortality HR for a hypoglycemic event in patients with type 2 diabetes is 2.69 .6 Hypoglycemia during hospital admissions is associated with increased lengths of stay and with increased 1-year mortality and inpatient mortality rates $(2.96 \%)$ for patients who had at least one hypoglycemic episode during the hospitalization vs. $0.82 \%$ for patients who had none [7]. Hypoglycemia and clinical outcomes in patients with diabetes hospitalized in the general ward. Diabetes Care. 2009; 32 (7); 1153-1157). Hypoglycemia can increase vascular inflammation, QT prolongation, intravascular coagulation, life threating arrythmias, and delayed clot thrombolysis. Therefore, patients with known cardiovascular disease must minimize their risk of hypoglycemia [8].

\section{Definition of Hypoglycemia}

The American Diabetes Association and the European Association for the Study of Diabetes (ADA/EASD) updated its standards of care and hypoglycemia definitions in 2017. (American Diabetes Association. Standards of medical care in diabetes. Diabetes Care. 2018;41 Suppl 1: S1-S159) The implications of different blood glucose levels vary from individual to individual but, $<70 \mathrm{mg} / \mathrm{dL}$ is considered an alert for hypoglycemia and allows patients time to take corrective action. A glucose level of $<54 \mathrm{mg} / \mathrm{dL}$ is considered clinically significant and unequivocally hypoglycemic. Severe hypoglycemia has no assigned biochemical value and is defined simply and starkly as a glucose level low enough to cause 
cognitive impairment such that the assistance of another person is required to administer carbohydrates or glucagon in order to achieve a recovery. Table 1 lists the American Diabetes Association definition of hypoglycemia levels [9].

Table 1: Basal Insulin Coefficient of Variability.

\begin{tabular}{|c|c|c|}
\hline Level & Definition & Glucose Value \\
\hline 1 & $\begin{array}{c}\text { Hypoglycemia alert } \\
\text { value (Take action) }\end{array}$ & $<70 \mathrm{mg} / \mathrm{dL}$ \\
\hline 2 & $\begin{array}{c}\text { Clinically significant } \\
\text { hypoglycemia }\end{array}$ & $\begin{array}{c}\text { No specific glucose threshold } \\
\text { hypoglycemia. Associated with severe } \\
\text { cognitive impairment requiring } \\
\text { external assistance for recovery }\end{array}$ \\
\hline 3 & Severe hypoglycemia \\
\hline
\end{tabular}

\section{Case Introduction-Tammy}

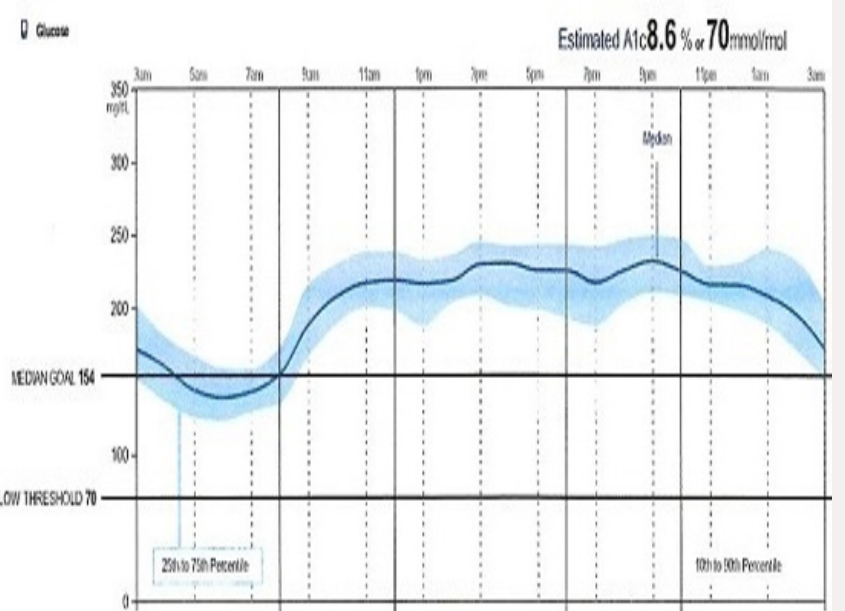

Figure 1a: 10 Day Continuous Glucose Sensor Report (Freestyle Libre).

Tammy is 68 years of age and has had poorly controlled type 2 diabetes. She is a former kindergarten teacher who neither smokes or drinks. Tammy had an inferior wall infarction a year ago after which she received 3 stents. She is adherent with her prescribed diabetes treatment regimen yet is unable to achieve her prescribed A1C target of $7.5 \%$. She is currently taking metformin 1 gram with breakfast and dinner, and liraglutide $1.8 \mathrm{mg} / \mathrm{d}$. Her blood pressure and lipids are at the American Diabetes Association recommended targets. Her A1C is $8.7 \%$. A download of her continuous glucose monitor (CGM) is shown in Figure 1a: The CGM begins recording interstitial glucose readings 12 hours after being inserted into the upper arm of the patient. A hand held "reader" is wanded across the sensor site providing the patient with a real-time glucose reading. The glucose level is stored in the reader and can be accessed in the form of charts, graphs, and data points. One important data set is the time within a prescribed targeted range (usually $80-180 \mathrm{mg} / \mathrm{dL}$ ) and the percent of time the patient is hypoglycemic $(<70 \mathrm{mg} / \mathrm{dL})$. The dark solid line provides graphic representation of the median interstitial glucose readings thorough out the day as well as during the life of the sensor (10 days). The blue zone which surrounds the median, represents the $25^{\text {th }}-75^{\text {th }}$ percentile in which $50 \%$ of the interstitial glucose levels are recorded. A separate 10-90\% curve represents the glucose levels of $90 \%$ of the glucose readings. This patient's glucose levels tend to drop overnight increasing Tammy's risk of experiencing unrecognized nocturnal hypoglycemia if she is intensively treated with basal insulin. Increased glycemic variability following dinner is also associated with hypoglycemia risk. The patient's sensor demonstrates persistent elevation in glucose levels throughout the day which correlates well with her A1C of $8.7 \%$. The clinician should consider intensifying her treatment with effective agents likely to allow the patient to achieve her targeted glycemic goals while minimizing the risk of hypoglycemia and weight gain.

\section{Case Continues}

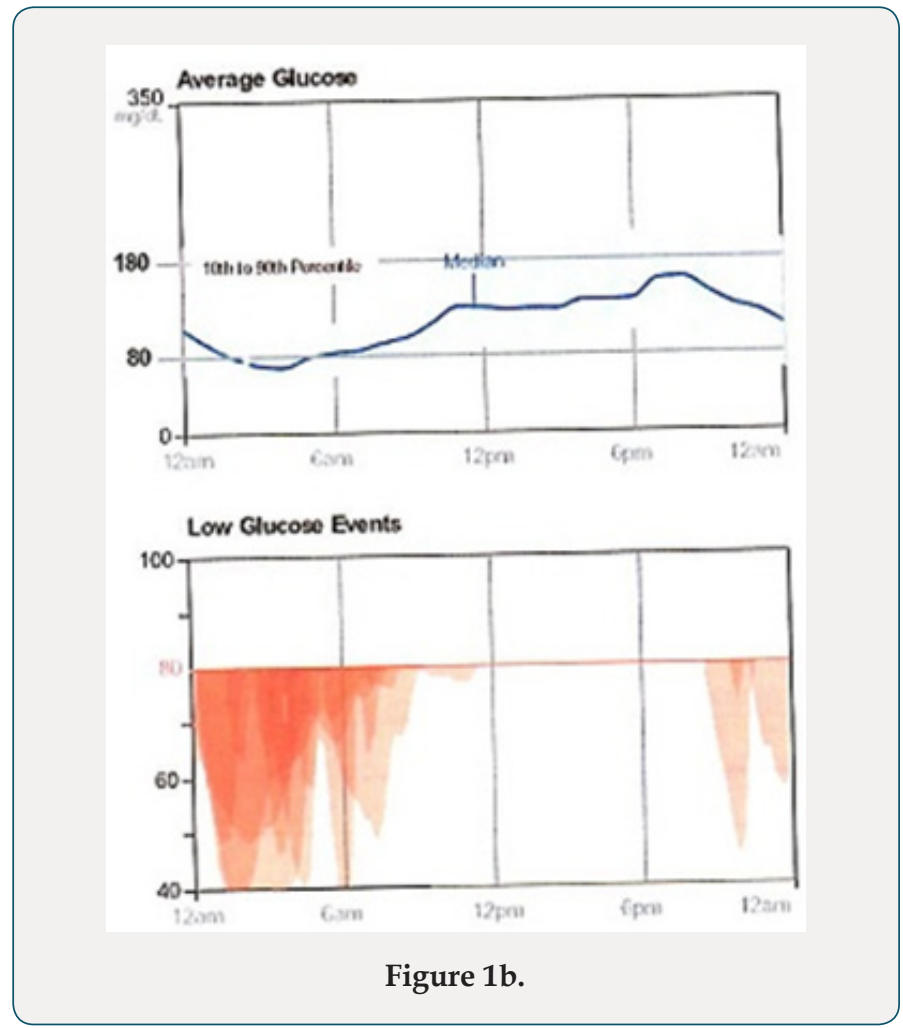

Tammy's physician prescribes insulin glargine to be used in conjunction with metformin 1 gram twice daily and liraglutide. The starting dose of glargine is 10 units at 9 PM daily. She is advised to increase the dose by 1 unit each night until her fasting blood glucose level is $<110 \mathrm{mg} / \mathrm{dL} .9$ The patient continues use of her CGM and titrates her insulin glargine as directed. Although her interstitial glucose levels improve, she is noted to have developed nocturnal hypoglycemia (Figure 1b). After titrating her insulin glargine dose from 10-32 units daily, our patient's glucose levels are noted to have dropped overnight resulting in unrecognized nocturnal hypoglycemia. In addition, her blood glucose levels tend to decline after dinner between the hours of 6 PM-12 AM. Tammy expresses relief that her glucose levels have improved but is concerned about the frequency of treatment induced nocturnal hypoglycemia. A1C, which is a reflection of one's average blood glucose levels over a 
90-day interval, is a poor predictor of hypoglycemia risk. Figure 2 shows the continuous glucose sensor readings of two insulin requiring patients with type 2 diabetes, both of whom have A1C levels of $7.8 \%$. Patient A (grey line) demonstrates erratic glucose readings ranging from 40 to $325 \mathrm{mg} / \mathrm{dL}$ (dysglycemia) whereas patient B (brown line) has less glycemic variability and no evidence of hypoglycemia. Intensifying the insulin regimen on patient A will result in severe hypoglycemia as well as weight gain and the induction of "oxidative stress" which could promote the development of long-term diabetes related complications [10]. Dysglycemia is shown in Figure 3. This can be a frustrating problem for patients as well as clinicians. CGM readings for a single patient taken over 4 separate days demonstrating significant glycemic variability occurring between the hours of 6-8 AM. High glycemic variability reduces one's quality of life and can promote mood disorders [11].

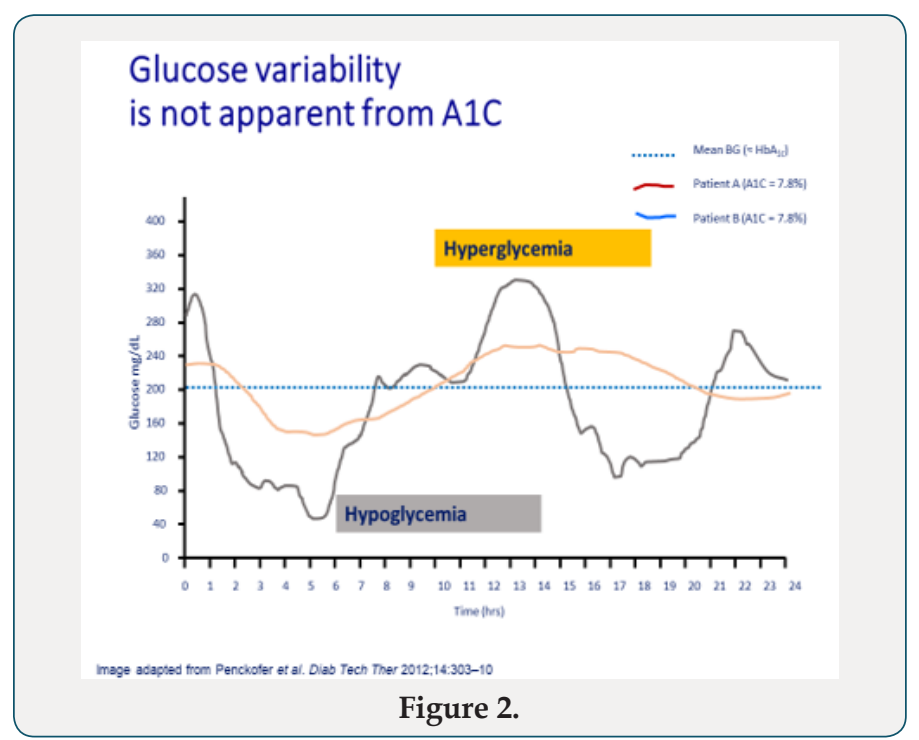

Glucose Variability Can Manifest as Fluctuating and Unpredictable Glucose Levels

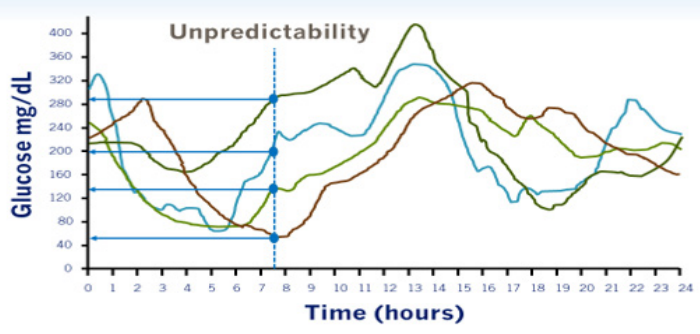

Figure 3: This can be a frustrating problem for patients as well as clinicians.

Patients who experience dysglycemia become frustrated with their inability to efficiently regulate blood glucose levels. The clinician may be asked, "I can't understand why on Tuesday my blood glucose level $46 \mathrm{mg} / \mathrm{dL}$ was, yet the following day I was at $278 \mathrm{mg} / \mathrm{dL}$ ?" All too often these patients are labeled as being "non-compliant" when, in fact, adherence to their prescribed pharmacologic regimen results in dysglycemia. Efficient pharmacotherapy for patients with diabetes must address both the effects of prolonged exposure to hyperglycemia as well as acute daily excursions of glucose levels which could increase one's risk of developing treatment emergent hypoglycemia Figure 4.

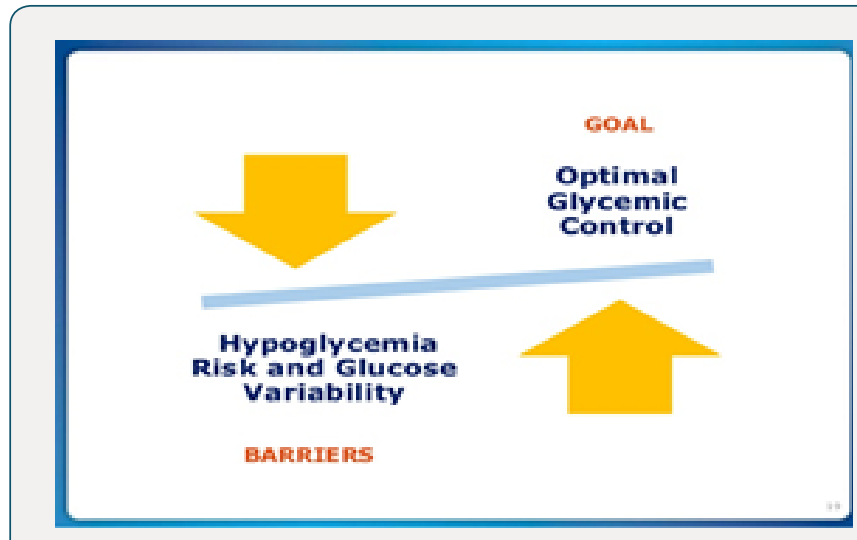

Figure 4: Hypoglycemia Risk Reduces One's Ability to Achieve Optimal and Targeted Glycemic Control.

\section{Addressing Glycemic Variability Using Novel Insulin Formulations}

Ideal basal insulins should be simple to initiate and titrate, resulting in minimal glycemic variability, as well as providing prolonged duration of action while reducing one's risk of hypoglycemia and weight gain. In addition, insulins should not increase one's risk of cardiovascular disease, especially in patients who have already experienced a stroke or myocardial infarction. Table 1 provides the coefficients of variability of available basal insulins. The lower the variability, the less likelihood of developing treatment-emergent hypoglycemia. Coefficient of variability is a predictor of hypoglycemia risk. The lower the coefficient, the less one is likely to experience hypoglycemia from that agent. Figure $5 a \& 5 b$ demonstrates the relationship between the coefficient of variability and hypoglycemia risk. Figure $5 c$ depicts ambulatory glucose monitoring (sensor data) from a patient with minimal glycemic variability. A patient is using an insulin with a high coefficient of variability. Glucose levels demonstrate "dysglycemia"

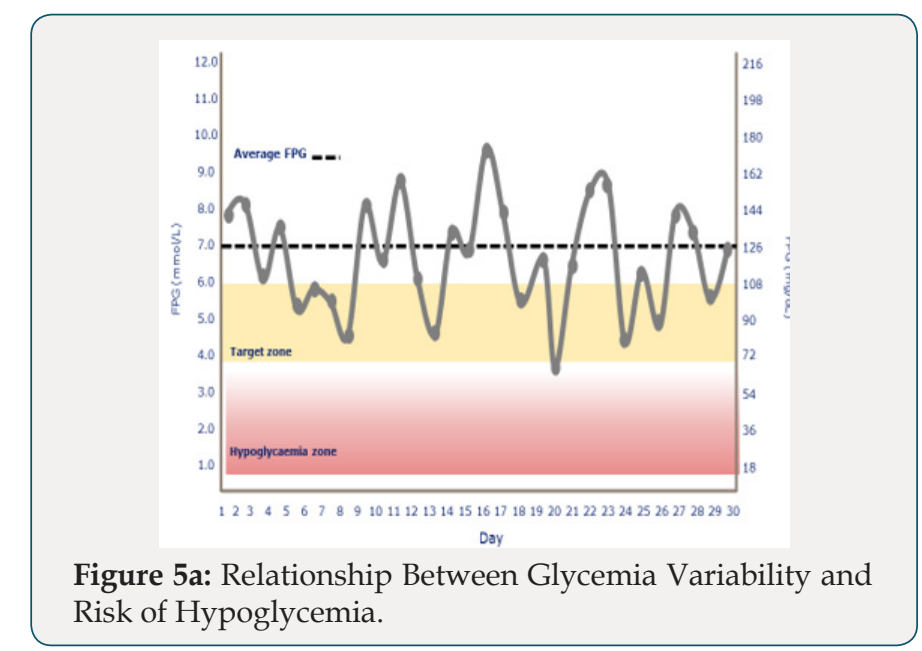




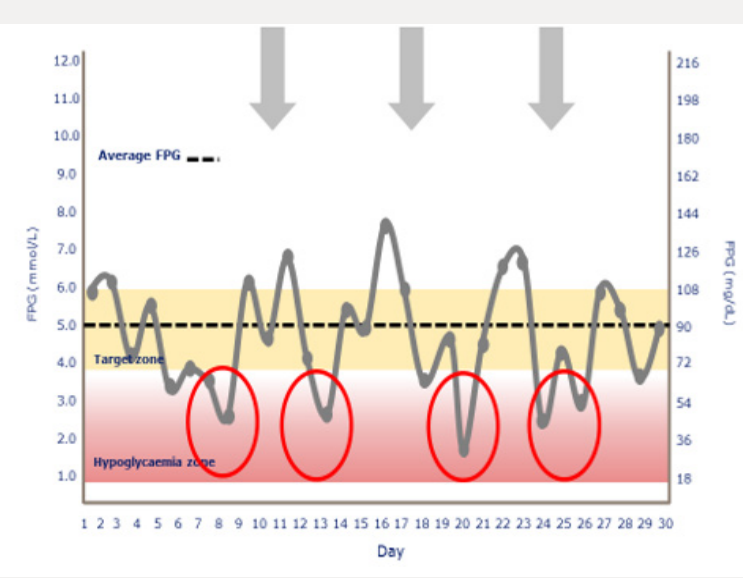

Figure 5b: Relationship Between Glycemia Variability and Risk of Hypoglycemia.

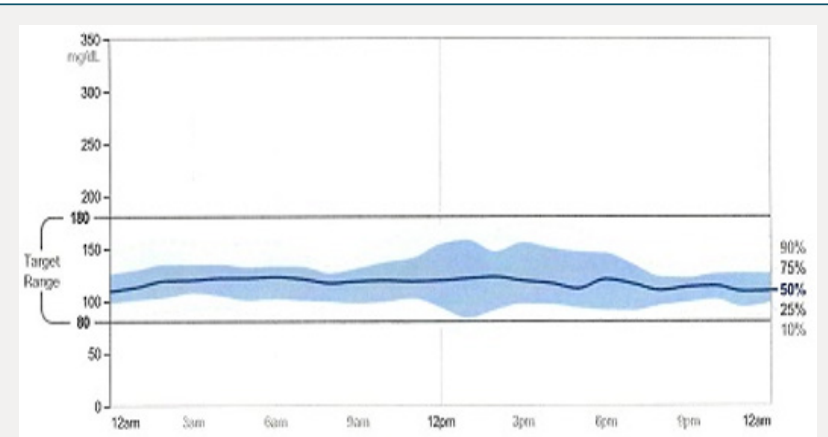

Figure 5c: Ambulatory blood glucose monitoring using the freestyle libre sensor demonstrating minimal glycemic variability with an essential flat 24 hour glucose profile (black line).

A. Increasing the basal insulin further is likely to increase the risk of hypoglycemia, which will reduce adherence to the prescribed treatment regimen

B. The use of basal insulin formulations with low glycemic variability will allow patients to achieve their fasting blood glucose targets more efficiently, with less fear of hypoglycemia [12]. Advances in Insulin Formulations.

Advances in basal insulin formulations have provided clinicians and patients with options that provide favorable pharmacokinetic (insulin absorption) and pharmacodynamic (glucose lowering) properties. Newer insulins have flatter, peak less action profiles that demonstrate less variability and a longer duration of action, allowing for flexible dosing. The risk of nocturnal and diurnal hypoglycemia is subsequently reduced. Insulin does not appear to increase cardiovascular risk [13]. Patients may also safely combine a GLP-1 RA as either a separate injection or as a component of a fixed-ratio drug. The use of fixed-drug combinations may improve adherence and allow patients to achieve their metabolic targets [14]. The insulin analogues glargine U100 and detemir have longer half-lives than neutral protamine Hagedorn (NPH) insulin, and at similar A1C levels reduce the frequency of overall and nocturnal hypoglycemia by $42-53 \%$. This reduction in hypoglycemia is most likely secondary to reduced day-to-day variability observed with these insulin analogues vs NPH [15]. Recently approved glargine U-300 and insulin degludec provide patients with a protracted duration of action while reducing the risk of confirmed and nocturnal hypoglycemia in patients with type 2 diabetes. Although insulin is recognized as the most effective blood glucose-lowering therapy, patients who experience hypoglycemia while on insulin are less likely to adhere to their prescribed insulin regimen thus reducing the likelihood of achieving their glycemic target [16]. Minimizing one's risk of experiencing treatment emergent hypoglycemia is imperative within the primary care practice model [17].

\section{Insulin Glargine U-300}

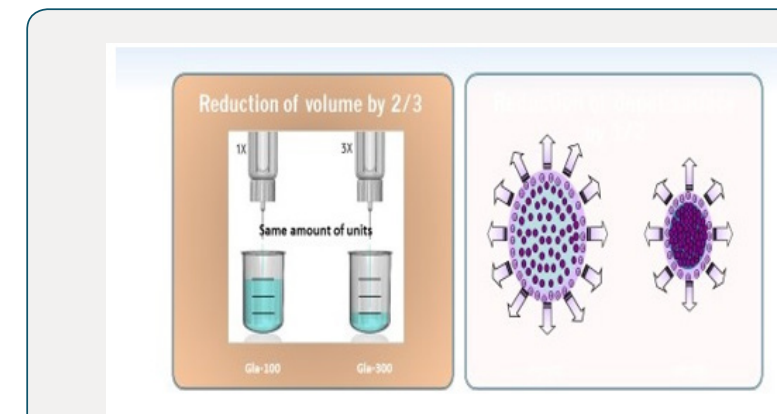

> Contains the same molecule as glargine (GLA100) but in a lower volume $>$ The decrease in volume decreases depot surface area, which can be predicted to slow down the rate of glargine release

Figure 6a: Graphic Comparison of Glargine U100 (Lantus) and Glargine U300 (Toujeo).

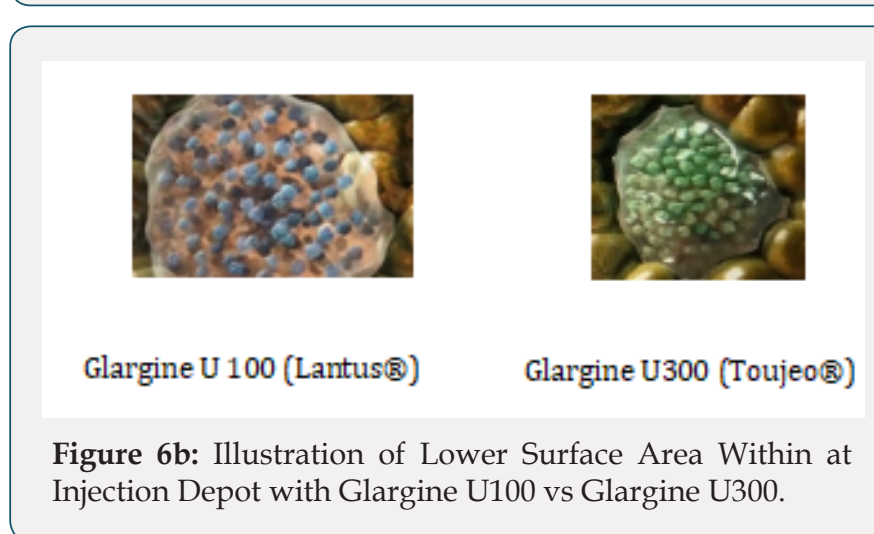

Glargine U-300 is a long-acting insulin containing 300 units/ $\mathrm{mL}$ of insulin glargine. U300 is soluble within the acidic $\mathrm{pH}$ of the injection medium (pen injector), but less soluble in the subcutaneous tissue once injected. Once exposed to the physiologic environment of the subcutis, the acidic PH of the pen's insulin solution forms a micro precipitate resulting in a slow and prolonged release of insulin from the injection depot. The glargine U300 molecule is identical to the amino acid sequence of U100 insulin. However, upon injection the more concentrated U300 forms a more compact subcutaneous depo with a smaller surface area leading to protraction of insulin absorption [18] (Figures 6a \& 6b). As a concentrated insulin, glargine U-300 contains 3 times as much insulin per $\mathrm{mL}$ as glargine U-100, allowing for a lower volume of injected insulin. Glargine 
U-300 was detectable at 32 hours post-injection with $0.4 \mathrm{units} / \mathrm{kg}$ dosing compared with 28 hours with glargine U100 dosing [19]. At $0.4 \mathrm{u} / \mathrm{kg}, \mathrm{U}-300$ has $14 \%$ less variability than U-100, allowing clinicians to titrate the insulin to target lower fasting glucose levels without risking hypoglycemia [20]. In a study comparing the two glargine insulin formulations, the risk of hypoglycemia was found to be $31 \%$ lower with glargine U300 compared with glargine U-100 owing to the superior pharmacokinetic profile of U300.21 (Figure 7) Thus, insulin requiring patients at risk for hypoglycemia could have $31 \%$ lower risk of hypoglycemia if initiated on glargine U300 instead of glargine U100. Note that both insulin formulations reduce $\mathrm{A} 1 \mathrm{C}$ equally at 6 months. However, the glargine U300 insulin reduces the risk of nocturnal hypoglycemia by $31 \%$ and also results in less weight gain than in patients taking glargine U100 [21].

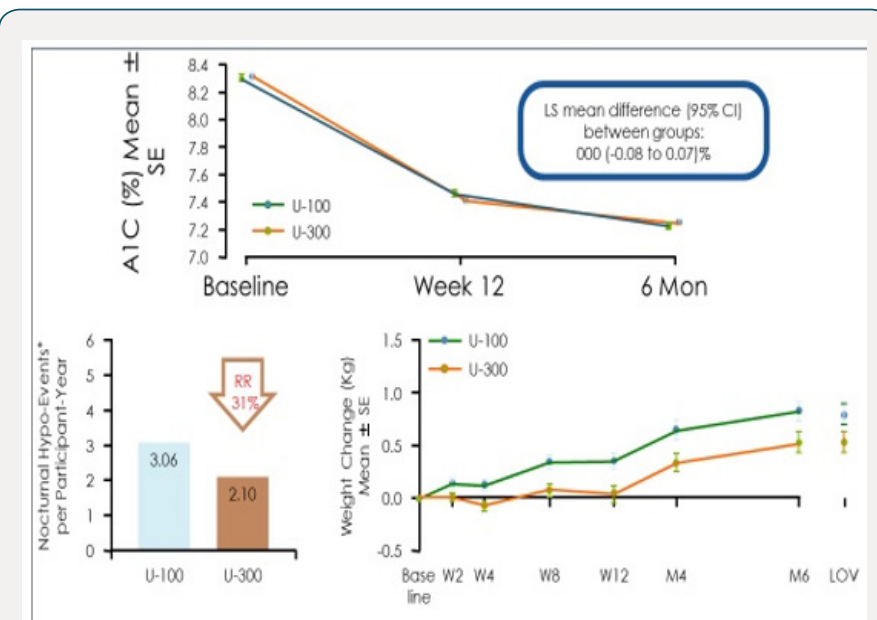

Figure 7: Safety and Efficacy of Glargine U300 vs. Glargine U100.

\section{Insulin Degludec}

Within the insulin pen, degludec is formulated as "insulin diheximers." Once injected, the diheximers form multiheximer chains within the subcutaneous depot, held together by zinc and phenol. As the zinc dissociates, the multiheximers form insulin monomers, which pass into the capillaries and are carried via albumin to insulin receptors at target organ sites. (Figure 8) Degludec U-200 contains as much insulin as degludec U-100 in just one-half the injection volume. The 2 degludec formulations are bioequivalent, and they lower glucose levels at the same rate. Degludec appears to have the lowest coefficient of variability of all insulins, allowing ambitious dosing to targeted fasting glucose levels, with less likelihood of nocturnal and overall hypoglycemia compared with insulin glargine [22]. Due to the prolonged duration of action (42 hours), degludec may be dosed at any time of the day, which may improve adherence for patients who are shift workers, travel frequently, or have difficulty remembering to dose their basal insulin [23]. Insulin degludec is maintained as diheximers within the insulin pen prior to injection. Once injected, the diheximers form multiheximers maintained by phenol and zinc. Over time, the phenol and zinc dissociate leaving the pharmacologically active form of insulin (monomers) to pass into the capillaries prior to targeting insulin receptor sites on cell membranes. Once the insulin binds to the receptor, glucose passes from the plasma into cells resulting in a reduction of blood glucose [24]. Insulin degludec and insulin glargine have demonstrated equivalent risk of treatment emergent cardiovascular events in patients with type 2 diabetes.

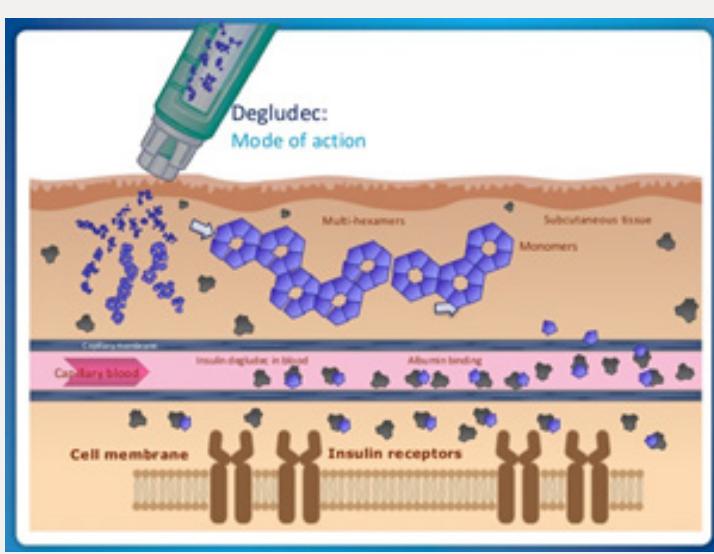

Figure 8: Insulin Degludec Mechanism of Protraction.

A randomized, double-blind, 2 period crossover, treat-to-target trial was conducted in patients with type 2 diabetes with basal insulin with or without oral antidiabetic drugs over 65 weeks.15 Patients were initiated on either insulin glargine or insulin degludec for 32 weeks followed by a blinded crossover to the other insulin. Each 32-week interval consisted of a 32-week titration period which allowed patients to achieve their glycemic targets (fasting glucose $71-90 \mathrm{mg} / \mathrm{dL}$ ), followed by a 16 -week maintenance phase comparing the difference in hypoglycemia events (confirmed blood glucose $<56 \mathrm{mg} / \mathrm{dL}$ or an episode requiring 3rd party assistance) when glycemic control and doses were stable. The primary endpoint was the rate of overall symptomatic hypoglycemic events or confirmed hypoglycemia during the 16-week maintenance phase. The secondary endpoints were the rate of nocturnal and symptomatic hypoglycemia occurring between the hours of 12:01 AM and 5:59 AM. The proportions of patients with hypoglycemic episodes were $22.5 \%$ for insulin degludec vs $31.6 \%$ for insulin glargine U100. The proportion of patients with nocturnal hypoglycemia events were $9.7 \%$ vs $14.7 \%$ favoring insulin degludec. $2.4 \%$ of patients using glargine U100 experienced severe hypoglycemia vs. $1.6 \%$ of patients using insulin degludec. Tammy is currently taking metformin 1-gram BiD, liraglutide $1.8 \mathrm{mg} / \mathrm{d}$ and insulin degludec 26 units once daily. Three months after initiating this regimen, Tammy's CGM download has provided the following graphics: Tammy's glycemic control has improved significantly. $83 \%$ of her interstitial glucose readings are within the target of $80-180 \mathrm{mg} / \mathrm{dl}$. However, $11 \%$ of her glucose levels are $<70 \mathrm{mg} / \mathrm{dL}$. These events appear to be occurring overnight as well as at 4 PM. 


\section{Targeting Hypoglycemia Prevention in High Risk Patients}

Improving patient education and empowering the patient to take some control over their disease are often very valuable tactics to improve both treatment outcomes, and treatment adherence, and therefore reduce the frequency of hypoglycemia. Patients prescribed drugs which work via a glucose independent manner (such as insulin and sulfonylureas) should be instructed to monitor for and report any treatment emergent hypoglycemia events. Adjustments in therapy will be required to mitigate risk. Patients who are unable to perceive the symptoms of hypoglycemia, should be prescribed continuous glucose sensors which alarm when a rapid decline in interstitial glucose levels are noted. Sensor will allow patients to act to reverse hypoglycemia before they develop cognitive impairment. Spouses and family members should be interviewed as well to determine if a patient has developed abnormal behaviors suggestive of hypoglycemia unawareness such as falls, confusion, disorientation, aggressive behavior, and nightmares (Tables $1 \& 2$ ). Family members should be instructed on the proper means of reversing severe hypoglycemia in patients with diabetes as shown in Figure 9 and Table 2 lists questions related to hypoglycemia which should be addressed at each patient visit.

Table 2: *Percentage within subject variability based on glucose infusion rates and area under the curve. Patients receive 4 single subcutaneous doses of $0.4 \mathrm{U} / \mathrm{kg}$ under euglycemic glucose clamp conditions on 4 study days. $\mathrm{NPH}=$ neutral protamine Hagedorn

\begin{tabular}{|c|c|}
\hline Insulin & Within-Subject Variability* \\
\hline NPH & 68 \\
\hline Glargine U-100 & 48 \\
\hline Detemir & 27 \\
\hline (Concentrated) Glargine U-300 & 34.8 \\
\hline Degludec & 20 \\
\hline
\end{tabular}

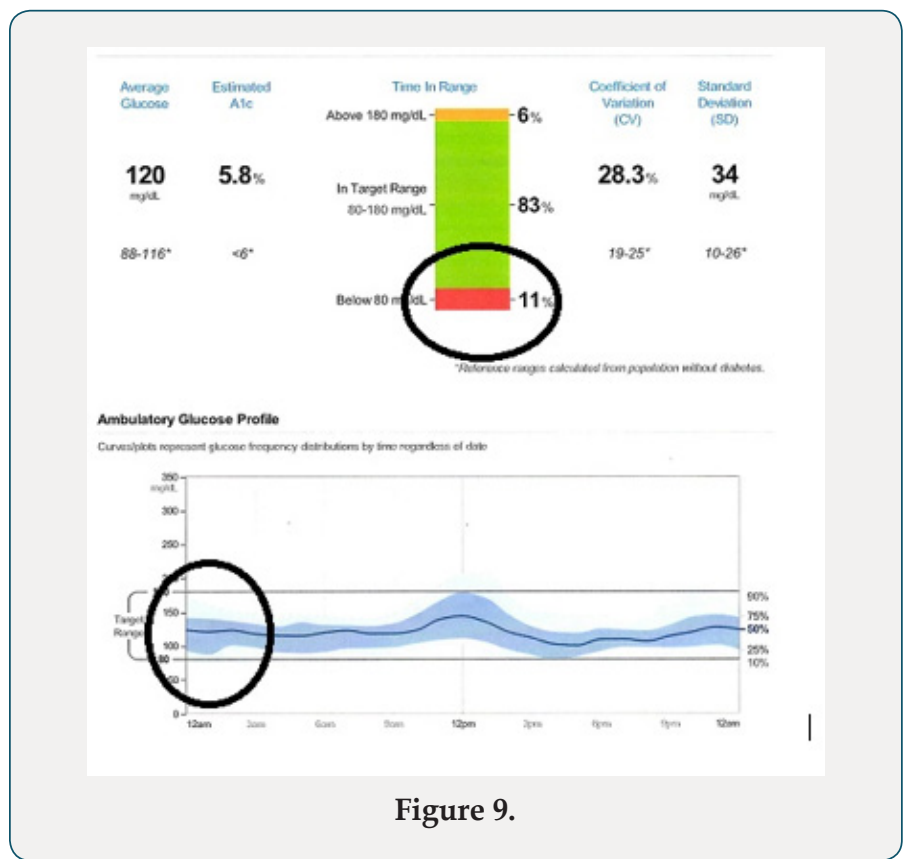

Table 2: Hypoglycemia Questions Which Should Be Addressed at Each Patient Visit

a) When did the hypoglycemic event occur (day time vs. nocturnal)?

b) Under what circumstances did they occur (missed meal, following exercise, excess medication)?

c) What were the symptoms?

d) What was the blood glucose or interstitial glucose reading?

e) How was the hypoglycemic event treated?

f) Did patient require assistance to reverse the hypoglycemia?

g) Did the hypoglycemic event recur within 24 hours?

h) How soon did the hypoglycemic event resolve with treatment?

i) How fearful is the patient regarding hypoglycemia events?

j) Does patient monitor glucose levels before driving?

k) As a result of the hypoglycemia, have you altered the dose of your diabetes medications?

l) Is the patient interested in obtaining a continuous glucose sensor which can predict and detect hypoglycemia before the patient becomes symptomatic?

Table 2 lists treatment recommendations for reversing hypoglycemia. Source: Adopted from: American Diabetes Association. http://www.diabetes.org/. Accessed 7/1/18.

Table 2: Management of Treatment Induced Hypoglycemia

A. Consume 15-20 grams of glucose or simple carbohydrates

i. Glucose tablets

ii. Gel tube

iii. "Glucose shot liquid"

iv. 2 tablespoons of raisins

v. $\quad 4 \mathrm{oz}(1 / 2$ cup) of juice or regular soda

vi. $\quad 8 \mathrm{oz}$ of nonfat or $1 \%$ milk

B. Recheck blood glucose after 15 minutes

C. If hypoglycemia persists, repeat 15-20grams of glucose

D. Once blood glucose normalized, eat a small snack if your next planned meal or snack is more than 2 hours away

E. Caregiver should inject unconscious patient with glucagon $1 \mathrm{mg}$ IM 
F. If glucagon is not available, place a teaspoon of honey in patient's mouth and sit them upright to avoid aspiration.

\section{Conclusion}

Iatrogenic hypoglycemia represents a much more substantial barrier to the effective control of blood glucose concentrations in patients with diabetes than is currently appreciated. Greater awareness and detection of all hypoglycemic events by careful monitoring, adherence to guidelines, and use of optimal treatment combinations is needed in order to prevent the serious medical and economic consequences associated with this adverse effect of antihyperglycemic medications. There is a need for improved patient and provider education on optimal detection and understanding of hypoglycemia and the benefits of accurate blood glucose measurement, which together can help to reduce the risk and fear of hypoglycemia, hypoglycemia unawareness, and hypoglycemic events. Therapeutic advances are continually being made, and insulins that ever more closely match the physiological profile of human insulin are currently FDA approved and should be considered as first line agents in patients at high risk for experiencing treatment emergent hypoglycemia. Patients may also consider use of continuous glucose monitoring and ambulatory glucose monitoring to mitigate their risk of hypoglycemia.

\section{References}

1. Zoungas S, Patel A, Chalmers J (2010) Severe hypoglycemia and risks of vascular events and death. N Engl J Med 363: 1410-1418.

2. Seshasai RK, Kaptoge S, Thompson A (2011) Diabetes mellitus, fasting glucose, and risk of cause-specific death. N Engl J Med 364: 829-884.

3. Chow E, Bernjak A, Walkinshaw E (2017) Cardiac autonomic regulation and repolarization during acute experimental hypoglycemia in type 2 diabetes. Diabetes 66 (5): 1322-1333.

4. Songer TJ, Dorsey RR (2006) High risk characteristics for motor vehicle crashes in persons with diabetes by age. Annu Proc Assoc Adv Automot Med 50: 335-351.

5. Zhang Y, Wieffer H, Modha R, Balar B, Pollack M, et al. (2010) The burden of hypoglycemia in type 2 diabetes: a systematic review of patient and economic perspectives. J Clin Outcomes Manag 17: 547-557.

6. Unger J (2013) Educating patients about hypoglycemia prevention and self-management. Clinical Diabetes 31(4): 179-188.

7. Desouza CV, Bolli GB, Fonseca V (2010) Hypoglycemia, diabetes, and cardiovascular events. Diabetes Care 33 (6): 1389-1394.

8. Gerstein HC, Yale JF, Harris SB (2006) A randomized trial of adding insulin glargine vs. avoidance of insulin in people with Type 2 diabetes on either no oral glucose-lowering agents or submaximal doses of metformin and/or sulphonylureas. The Canadian INSIGHT (Implementing New Strategies with Insulin Glargine for Hyperglycaemia Treatment) Study. Diabet Med 23(7): 736-742.
9. Unger J (2008) Reducing oxidative stress in patients with type 2 diabetes mellitus: A Primary Care call to action. Insulin 3: 176-184.

10. Penckofer S, Quinn L, Byrn M (2010) Does glycemic variability impact mood and quality of life? Diabetes Tech and Ther 14(4): 303-310.

11. Unger J (2018) Concentrated and Fixed-Dose Insulin Formulations Can Improve Outcomes in Patients with Type 2 Diabetes Mellitus. Endocrinology, Diabetes, Metabolism Journal 3(1).

12. Gerstein HC, Bosch J, Dagenais GR, Díaz R, Jung H, et al. (2012) Basal insulin and cardiovascular and other outcomes in dysglycemia. $\mathrm{N} \mathrm{Engl} \mathrm{J}$ Med 367(4): 319-328.

13. Ferdinand KC, Senatore FF, Clayton-Jeter H (2017) Improving medication adherence in cardiometabolic disease: practical and regulatory implications. J Am Coll Cardiol 69(4): 437-451.

14. Wysham C, Bhargava B, Norwood P (2017) Effect of insulin degludec vs insulin glargine U100 on hypoglycemia in patients with type 2 diabetes. JAMA 318(1): 45-56.

15. Edelman SV, Polonsky WH (2017) Type 2 diabetes in the real world: the elusive nature of glycemic control. Diabetes Care 40: 1425-1432.

16. Unger J (2018) A clinical overview of once weekly glucagon-like peptide 1 receptor agonists. Journal of Family Practice 67(6): S1-S2.

17. Riddle MC, Boli GB, Zieman M (2014) New insulin glargine 300 units/mL versus glargine 100 units $/ \mathrm{mL}$ in people with type 2 diabetes using basal and mealtime insulin: glucose control and hypoglycemia in a 6-month randomized controlled trial ( $1^{\text {st }}$ Edn). Diabetes Care 37(10): 2755-2762.

18. Becker RH, Dahmen R, Bergmann K, Lehmann A, Jax T, et al. (2015) New insulin glargine 300 Units. $\mathrm{mL}-1$ provides a more even activity profile and prolonged glycemic control at steady state compared with insulin glargine 100 Units. mL-1. Diabetes Care 38(4): 637-643.

19. Becker RH, Nowotny I, Teichert L, Bergmann K, Kapitza C (2015) Low within- and between-day variability in exposure to new insulin glargine 300U/ml. Diabetes Obes Metab 17(3): 261-267.

20. Ritzel R, Roussel R, Bolli GB (2015) Patient-level meta-analysis of the EDITION 1,2 and 3 studies. Glycaemic control and hypoglycemia with new insulin glargine $300 \mathrm{U} / \mathrm{mL}$ versus glargine $100 \mathrm{U} / \mathrm{ml}$ in people with type 2 diabetes. Diabetes, Obes Metab 17(9): 859-867.

21. Wysham C, Bhargava A, Chaykin L (2017) Effect of insulin degludec vs insulin glargine U100 on hypoglycemia in patients with type 2 diabetes The SWITCH 2 randomized clinical trial. JAMA 318(1): 45-56.

22. Meneghini L, Atkin SL, Gough SC (2013) The efficacy and safety of insulin degludec given in variable once-daily dosing intervals compared with insulin glargine and insulin degludec dosed at the same time daily; a 26-week, randomized, open-label, parallel-group, treat-to-target trial in individuals with type 2 diabetes. Diabetes Care 36(4): 858-864.

23. Haahr H, Heise T (2014) A review of the pharmacologic properties of insulin degludec and their clinical relevance. Clin Pharmacokinet 53(9): 787-800.

24. Marso SP, McGuire DK, Zinman B (2017) Efficacy and safety of degludec versus glargine in type 2 diabetes. $\mathrm{N}$ Engl J Med 377(8): 723-732. 
(C) (i) This work is licensed under Creative To Submit Your Article Click Here:

Submit Article

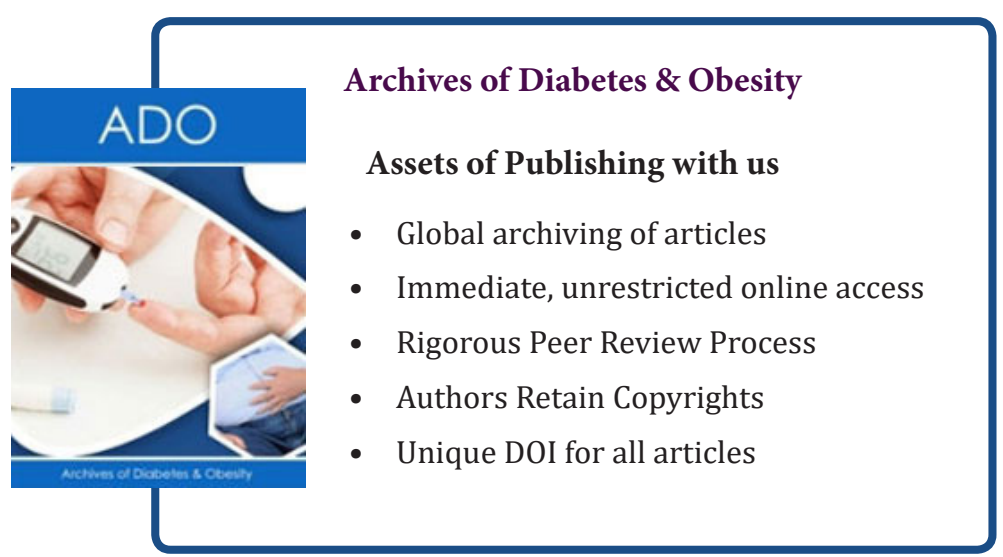

